\title{
Editorial
}

\section{Temporomandibular disorders}

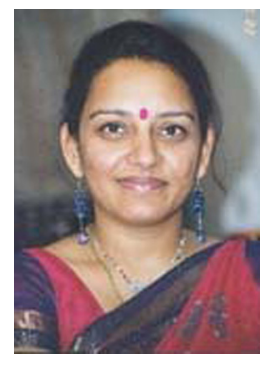

Temporomandibular joint, unlike other joints in the human body, is different in terms of its anatomy and management of ailments. Proficiency in the management of TMJ disorders has always created epoch among oral and maxillofacial surgeons worldwide. Due to lifestyle changes, TMJ pain has emerged as a big nuisance crippling our society.

American Dental Association's conference on temporomandibular disorders (TMDs) in 1983, discussed and highlighted the need for a reliable and valid diagnostic classification system to identify TMD and their specific subtypes. ${ }^{1}$ National Institutes of Health Technology Assessment Conference Statement, 1996 specified the need to identify the pathogenesis and risk factors for TMDs through epidemiological and experimental studies. ${ }^{2}$ Such studies have provided the basis for an etiology-based diagnostic classification system necessary to best facilitate management and treatments for these disorders.

The Research Diagnostic Criteria for Temporomandibular Disorders (RDC/TMD) presented by Dworkin and LeResche, 1992, defines TMD cases and their specific subtypes. ${ }^{3}$ The $\mathrm{RDC} / \mathrm{TMD}$ has been used in many epidemiologic and clinical studies of TMDs. This system includes two Axis; I (clinical TMD conditions) physical assessment and diagnostic protocol, and II (pain-related disability and psychological status) assessment of psychological status and pain-related disability. TMD pain should be distinguished from other pain conditions, such as referred pain, hyperalgesia, allodynia and central sensitization in the masticatory.

Keeping this in mind, TMJ Conference \& Mastercourse 2013 was conducted in Lucknow from 22nd to 24th Aug' 2013. The whole scientific program revived and refreshed our minds with the knowledge of temporomandibular joint afflictions and its management. The 2 days program gave us a broad overview of varied topics ranging from the surgical anatomy of and approaches to TMJ, Myofascial and TMJ pain, arthritis and internal disc derangements, recurrent dislocations and ankylosis, diagnostic advancements such as MRIs, arthroscopy, non surgical treatment modalities, minimal invasive arthrocentesis and open joint surgery, rehabilitation of TMJ with TM joint replacement or reconstruction using various alloplastic \& autogenous materials, correction of post ankylotic deformities and distraction osteogenesis, and biomedical tissue engineering.

This issue contains the topics discussed by experts in the field and abstracts of various papers presented during the conference.

\section{R E F E R E N C E S}

1. Laskin D, Greenfield W, Gale E, et al., eds. The President's Conference on the Examination, Diagnosis and Management of Temporomandibular Disorders. Chicago: American Dental Association; 1983.

2. Management of temporomandibular disorders. National Institutes of Health Technology Assessment Conference Statement. J Am Dent Assoc. 1996;127:1595-1606.

3. Dworkin SF, LeResche L, eds. Research Diagnostic Criteria for Temporomandibular Disorders: Review, Criteria, Examinations and Specifications, Critique. J Craniomandib Disord. 1992; vol. 6(4):301-355.

Divya Mehrotra

Prof, Editor in Chief, Department of Oral \& Maxillofacial Surgery, King George Medical University, Lucknow, Uttar Pradesh, India E-mail address: divyamehrotra@hotmail.com

2212-4268/\$ - see front matter Copyright $\odot 2013$, Craniofacial Research Foundation. All rights reserved. http://dx.doi.org/10.1016/j.jobcr.2013.10.002 